\title{
Biophysical Properties of HP1-Mediated Heterochromatin
}

\author{
Serena Sanulli, ${ }^{1}$ John D. Gross,${ }^{1}$ and Geeta J. Narlikar ${ }^{2}$ \\ ${ }^{1}$ Department of Pharmaceutical Chemistry, \\ ${ }^{2}$ Department of Biochemistry and Biophysics, University of California, \\ San Francisco, California 94158, USA \\ Correspondence: geeta.narlikar@ucsf.edu
}

\begin{abstract}
Heterochromatin is a classic context for studying the mechanisms of chromatin organization. At the core of a highly conserved type of heterochromatin is the complex formed between chromatin methylated on histone H3 lysine 9 and HP1 proteins. This type of heterochromatin plays central roles in gene repression, genome stability, and nuclear mechanics. Systematic studies over the last several decades have provided insight into the biophysical mechanisms by which the HP1-chromatin complex is formed. Here, we discuss these studies together with recent findings indicating a role for phase separation in heterochromatin organization and function. We suggest that the different functions of HP1-mediated heterochromatin may rely on the increasing diversity being uncovered in the biophysical properties of HP1-chromatin complexes.
\end{abstract}

Packaging of the eukaryotic genome into chromatin provides the first level of gene regulation by occlusion of specific DNA regions from the transcription machinery (Luger et al. 1997; Jost et al. 2012). The second level of regulation is achieved by the organization of chromatin into transcriptionally active regions called euchromatin and transcriptionally silent regions called heterochromatin (Grewal and Jia 2007; Allshire and Madhani 2018). These two levels of regulation play central roles in the determination of cellular identity and function. The term heterochromatin was originally used by Heitz (1928) to describe regions of chromosomes that appeared to remain condensed throughout the cell cycle (Heitz 1928; Jost et al. 2012). Pioneering genetic and biochemical work by Elgin and colleagues showed that a central component of heterochromatin was the protein HP1, which could bind chromatin that was methylated on histone H3 lysine 9 (James and Elgin 1986; James et al. 1989; Eissenberg et al. 1990). These findings were followed by the discovery of the enzyme responsible for $\mathrm{H} 3 \mathrm{~K} 9$ methylation by Jenuwein and colleagues (Rea et al. 2000). HP1-mediated heterochromatin is found in several organisms including yeast and humans. Another type of heterochromatin that is also found in multiple organisms is mediated by the Polycomb group of proteins (Simon and Kingston 2009; Schuettengruber et al. 2017; Yu et al. 2019). These proteins act in large complexes and are commonly associated with chromatin that is methylated on histone H3 lysine 27. In this perspective, we will focus on HP1-mediated heterochromatin.

HP1-mediated heterochromatin is often functionally classified into two types: constitutive heterochromatin, which is found near centromeres and telomeres and appears largely invariant with cell type; and facultative heterochromatin, which enables repression of developmentally controlled genes and is cell type-specific (Allshire and
Madhani 2018; Nicetto and Zaret 2019). Gene repression is thought to arise from at least two mutually compatible mechanisms: steric occlusion by HP1 binding and chromatin compaction, and recruitment of gene silencing machineries via noncoding RNA based mechanisms (Grewal and Jia 2007; Allshire and Madhani 2018). In this review, we do not discuss the noncoding RNA based pathways and instead refer the reader to some excellent reviews on this topic (Reyes-Turcu and Grewal 2012; Martienssen and Moazed 2015; Allshire and Madhani 2018).

Work from several groups has shown that heterochromatin mediated by HP1 plays other roles in addition to gene repression. One of these roles is enabling correct chromosome segregation (Allshire and Madhani 2018; Janssen et al. 2018). It is proposed that centromeric heterochromatin provides a critical platform for regulating the deposition of the centromeric histone $\mathrm{H} 3$ and for regulating cohesion action (Allshire and Madhani 2018). Another role involves providing mechanical stability to cells during interphase (Stephens et al. 2019). Such a role for HP1-mediated heterochromatin is proposed to arise in part from the ability of HP1 to interact with nuclear lamin proteins that provide much of the structural integrity to the nuclear envelope (Stephens et al. 2019). Thus HP1heterochromatin functions across multiple scales: from the scale of individual genes to the scale of the whole nucleus.

A central and common feature in almost all of these functions is the HP1-chromatin complex. We and others have been focused on understanding the biophysical capabilities of this complex. Below we first summarize prior advances in this area and then describe recent data from our laboratories on the linkage between phase separation by HP1 proteins and chromatin compaction. Finally, we conclude with some speculations on the biophysical basis for the diverse functions performed by heterochromatin.

(C) 2019 Sanulli et al. This article is distributed under the terms of the Creative Commons Attribution-NonCommercial License, which permits reuse and redistribution, except for commercial purposes, provided that the original author and source are credited. 


\section{SANULLI ET AL.}

\section{OLIGOMERIZATION BEHAVIOR OF HP1 PROTEINS}

Early studies in Drosophila found that transposition of a gene from euchromatin into heterochromatin resulted in the silencing of the gene in some cells but not others (Elgin and Reuter 2013). This phenomenon termed position effect variegation was attributed to the spreading of heterochromatic material over the transposed gene. Later studies found that such gene silencing was accompanied by the presence of the H3K9me3 mark and HP1 over the silenced gene (Elgin and Reuter 2013). Therefore, one property attributed to heterochromatin has been the ability to spread across large regions of the genome. From a biophysical perspective, the phenomenon of "spreading" suggests some manner of cooperative action by heterochromatin molecules on chromatin. In this context, as summarized below HP1 molecules can both dimerize and form high-order oligomers.

The HP1 molecule contains two structured domains, the chromodomain (CD) that binds the $\mathrm{H} 3 \mathrm{~K} 9$ me3 mark and the chromoshadow domain (CSD) that forms a homodimer (Canzio et al. 2014; Eissenberg and Elgin 2014) (Fig. 1A). In addition, there are three unstructured regions, the hinge (H), which binds both DNA and RNA, an amino-terminal extension (NTE), and a carboxy-terminal extension (CTE) (Sugimoto et al. 1996; Muchardt et al. 2002; Meehan et al. 2003; Mishima et al. 2013). Cross-linking studies with isolated CD and CSD domains of the human HP $1 \alpha$ protein helped uncover the potential of these domains to form higher-order structures (Yamada et al. 1999). High-resolution crystal structures of CSD-CSD dimers from various HP1 paralogs provided a detailed view into the dimerization interface and further indicated how the CSD-CSD dimer could interact with other protein ligands (Brasher et al. 2000; Cowieson et al. 2000; Smothers and Henikoff 2000; Thiru et al. 2004; Kang et al. 2011; Mendez et al. 2013). Overall, most HP1 proteins studied were found to be able to form dimers, although the dissociation constants ranged from low $\mathrm{nM}$ to $\mu \mathrm{M}$ (Canzio et al. 2014).

Gel-filtration studies with the major Schizosaccharomyces pombe HP1 protein Swi6 first raised the possibility

A

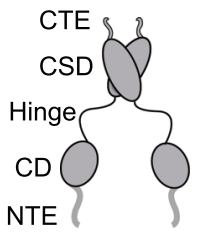

\section{S. pombe Mammals (mouse and human)}

Swi6, Chp2 HP1 $1 \alpha, \operatorname{HP} 1 \beta, \mathrm{HP} 1 \gamma$

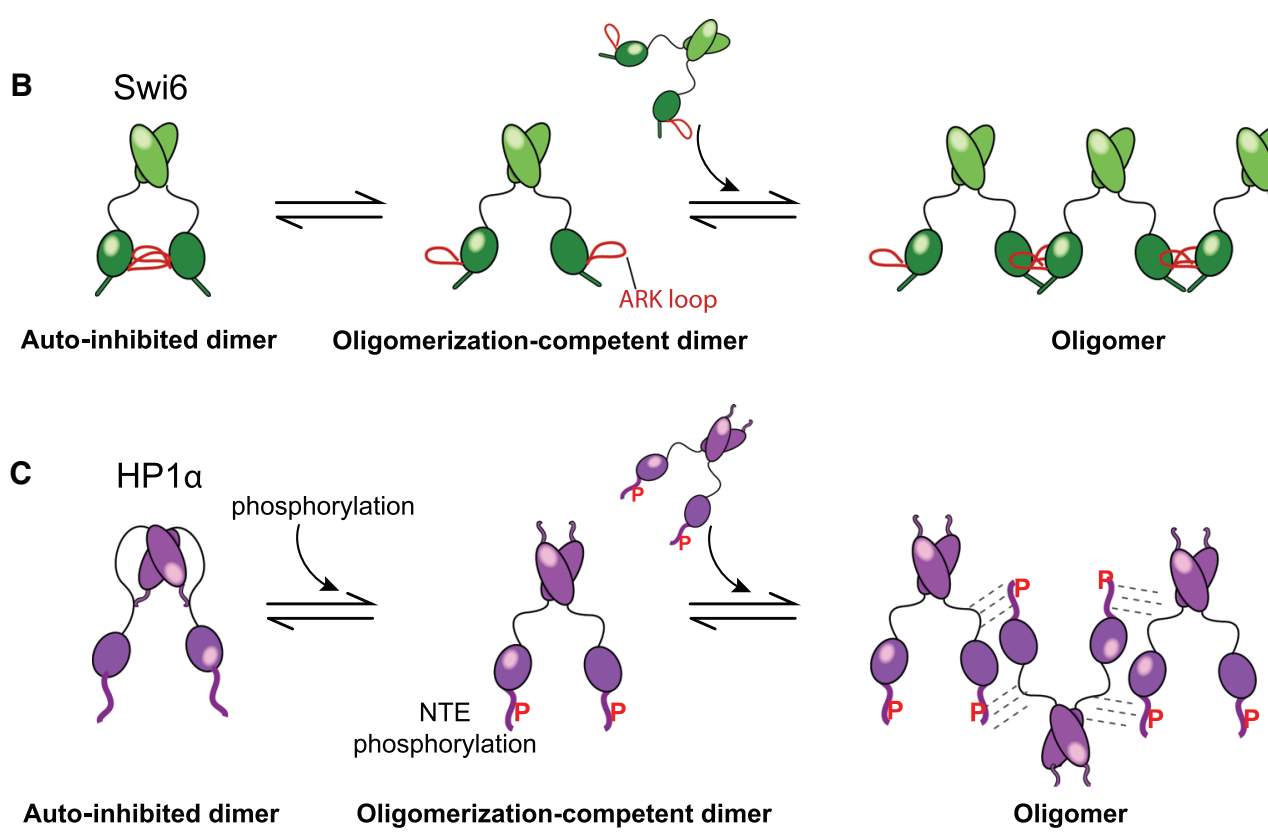

Figure 1. Comparison of HP1 molecules between Schizosaccharomyces pombe and mouse and human. (A) (Left) Domain architecture found in yeast and mammalian HP1 proteins. (Right) Nomeclature of HP1 proteins studied biochemically. S. pombe has two HP1 proteins, Swi6 and Chp2, whereas mouse and humans have three HP1 proteins that have been studied biochemically, HP1 $\alpha, \operatorname{Hp} 1 \beta$, and HP1 $\gamma .(B)$ Model showing auto-inhibition in Swi6 mediated by the interaction between the ARK loop in red and the chromodomain binding pocket in green. The ARK loop also participates in stabilizing higher-order oligomers by interacting with the CD of a different dimer. $(C)$ Model showing auto-inhibition in HP1 $\alpha$ mediated by the interaction between the CTE and the hinge. Phosphorylation of the NTE relieves the auto-inhibition and promotes oligomerization via NTE-hinge interactions mediated by the phosphate groups with positively charged hinge residues. The phosphate groups are shown by the red "P" letter. 
that the full-length proteins may form oligomers beyond dimers (Wang et al. 2000). Later studies indicated that the gel-filtration results largely reflected a dimeric state of Swi6 (Sadaie et al. 2008). The possibility of higher-order oligomerization was conclusively tested by analytical ultracentrifugation, which enabled quantification of the true masses of the Swi6 oligomers (Canzio et al. 2011; Canzio et al. 2013). These experiments showed that Swi6 could form higher-order oligomers by sequential addition of dimers. Interestingly, Swi6 dimers were found to exist in an auto-inhibited state, and switching out of the auto-inhibited state was required to form higher-order oligomers (Canzio et al. 2013). Auto-inhibition arises because the $\mathrm{CD}$ of one monomer within a Swi6 dimer is bound by a loop present in the $\mathrm{CD}$ of the other monomer (Fig. 1B). The loop sequence (ARK) mimics the H3 tail sequence that is bound by the CD. Further, the interactions formed in the oligomeric state also appear to involve the CDARK loop interaction, but between two dimers rather than within a dimer. Studies performed with the human HP1 $\alpha$ protein found that phosphorylation of the NTE (nPhos_HP1 $\alpha$ ) was essential to switch the protein from an auto-inhibited state to an oligomerization competent state (Fig. 1C) (Larson et al. 2017). In this case, autoinhibition is thought to be enabled by the interaction of the CTE of one monomer with the hinge of the second monomer. This model for auto-inhibition is consistent with earlier studies suggesting that the CSD region plays an auto-inhibitory role to regulate the ability of the hinge to bind DNA (Mishima et al. 2013). The oligomeric state of nPhos_HP1 $\alpha$ was suggested to involve interactions between two of its unstructured regions, the hinge and the NTE (Larson et al. 2017). Thus, analogous to the case with Swi6, common interaction interfaces are used in the auto-inhibited and oligomeric states (Fig. 1B,C).

Overall the studies above provided a key conceptual building block for understanding the biophysical basis of heterochromatin function: the ability of HP1 proteins to form higher-order oligomers in an autoregulated manner.

At the same time, these studies also uncovered interesting differences between HP1 paralogs. For example, although human HP1 $\alpha$ formed higher-order oligomers upon phosphorylation, its paralog HP1 $\beta$ did not (Larson et al. 2017). How the sequence differences between HP1 $\alpha$ and HP1 $\beta$ contribute to these differences in biophysical behavior is an active area of research, and some additional biophysical differences are mentioned in the sections below.

\section{HP1 INTERACTION WITH CHROMATIN}

Several studies have characterized the biophysical behavior of HP1 proteins on chromatin. Early studies have shown that the CD of HP1 proteins can bind the H3K9me3 mark with high specificity (Bannister et al. 2001; Jacobs et al. 2001; Lachner et al. 2001; Jacobs and Khorasanizadeh 2002; Nielsen et al. 2002). Further, aminoterminal phosphorylation of mammalian HP1 $\alpha$ has been shown to increase the affinity of its CD for the H3K9me3 mark (Hiragami-Hamada et al. 2011; Nishibuchi et al.
2014). Work with full-length Swi6 has found that four molecules of Swi6 bind one nucleosome (Canzio et al. 2011). Within the Swi6-nucleosome complex, the CSD-CSD dimer was implicated in binding the nucleosome core (Canzio et al. 2013). Later studies, discussed in detail further below, indicated that the CSD-CSD dimer contacts the $\alpha 1$-helix of H2B (Sanulli et al. 2019) (Fig. 2C). Interestingly, the ARK loop within the CD that participates in auto-inhibition and oligomerization was also implicated in binding nucleosomal DNA (Fig. 2A) (Canzio et al. 2013). Together, these findings led to a model where the two unbound CDs act as "sticky ends" that interact with either nearby CDs or nearby nucleosomes (Fig. 2D). In the former possibility, bridging requires both the dimerization and a tetramerization interfaces. This possibility is consistent with and builds on previous models invoking a tetrameric Swi6 complex (Wang et al. 2000). The latter possibility relies mainly on the dimerization interface. Consistent with a nucleosome bridging role, Swi6 was shown to bind with higher specificity for the H3K9me3 mark on nucleosomal arrays with $15 \mathrm{bp}$ versus $47 \mathrm{bp}$ of internucleosomal spacing (Canzio et al. 2011).

Studies with mammalian HP1 $\beta$ have suggested a nucleosome bridging model that largely relies on the HP1 dimer interface (Hiragami-Hamada et al. 2016) (Fig. 2D). Studies with mammalian $\mathrm{HP} 1 \alpha$ have shown that $\mathrm{HP} 1 \alpha$ can bind nucleosome arrays and compact them (Fig. 2B) (Azzaz et al. 2014; Kilic et al. 2018). Further, EM studies have suggested that one HP1 $\alpha$ dimer can bridge two nucleosomes (Machida et al. 2018). However, whether HP1 $\beta$ and HP1 $\alpha$ can adopt other stoichiometries on nucleosomes is not known. This is because unlike with Swi6 the stoichiometry of these proteins on mononucleosomes in solution has not been directly measured.

In terms of contacting the histone core, the CSD-CSD interface of mammalian HP1 proteins has been shown to interact with the $\mathrm{H} 3 \alpha \mathrm{N}$ helix region of the nucleosome core (Fig. 2C) (Dawson et al. 2009; Lavigne et al. 2009; Richart et al. 2012). The different regions of the octamer contacted by mammalian HP1 proteins versus Swi6 are consistent with the different binding specificities identified for their respective CSD-CSD dimers (Fig. 2C) (Smothers and Henikoff 2000; Thiru et al. 2004; Lechner et al. 2005; Mendez et al. 2011; Mendez et al. 2013; Isaac et al. 2017).

Interestingly, phosphorylation of the NTE of mammalian HP $1 \alpha$ has been suggested to enhance its specificity for the H3K9me3 mark within nucleosomes while decreasing its affinity for nucleosomes and DNA (Hiragami-Hamada et al. 2011). These findings raise the possibility that phosphorylation dissociates HP1 $\alpha$ from the nucleosome core such that it is now bound mainly to the $\mathrm{H} 3$ tail.

Overall the studies in this and the prior section have showcased the diverse mechanisms by which HP1 proteins could assemble on chromatin and enable chromatin compaction.

\section{PHASE SEPARATION OF HP1 PROTEINS TO EXPLAIN COMPARTMENTALIZATION}

In 2017, two studies reported the ability of HP1 proteins to form phase-separated droplets (Larson et al. 2017; 
A

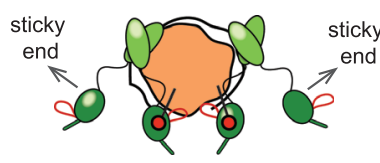

Swi6-nucleosome complex
B

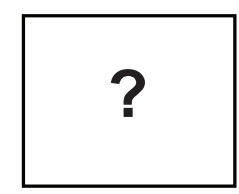

HP1 $\alpha$-nucleosome complex
C
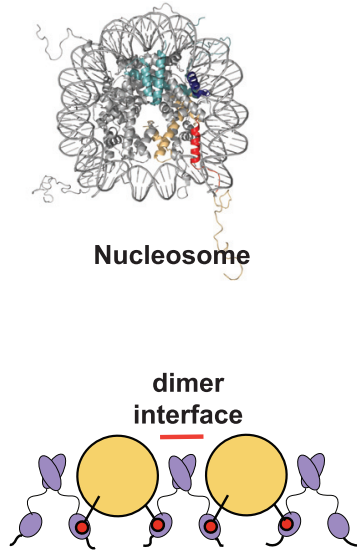

D

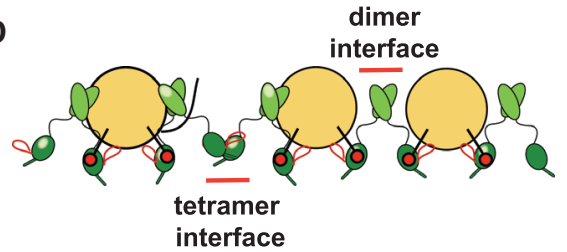

Figure 2. Nucleosomal interactions made by HP1 proteins. (A) Model for Swi6 interaction with a mononucleosome. Binding of Swi6 deforms the nucleosome core. The ARK loop on the CD is in red, deformed histone octamer is in orange, loosened DNA is in black, and the H3K9 methylation mark is shown as a red circle on the $\mathrm{H} 3$ tail, which is in black. $(B)$ The stoichiometry of mammalian HP1 $\alpha$ on nucleosomes in solution has not been measured. $(C)$ The crystal structure of the nucleosome is shown with the DNA, histone H4, and histone $\mathrm{H} 2 \mathrm{~A}$ in gray. One copy each of histone $\mathrm{H} 3$ and $\mathrm{H} 2 \mathrm{~B}$ are shown in orange and blue, respectively. The histone helices that are suggested to be contacted by the CSD dimers of Swi6 and the mammalian HP1 proteins, respectively, are shown in dark orange (in H3) and dark blue (in H2B), respectively. $(D)$ Two modes of nucleosome bridging. (Left) Model for two modes by which Swi6 can bridge across nucleosomes. The tetramerization interface between two dimers facilitates bridging. The tetramerization interface is stabilized by the CD-ARK loop interaction. (Right) Model for how HP1 $\beta$ may bridge nucleosomes. The dimer interface facilitates bridging. The two nucleosomes shown could be adjacent nucleosomes on the same chromatin fiber, nonadjacent nucleosomes on the same chromatin fiber, or nucleosomes on two different chromatin fibers.

Strom et al. 2017). One study showed that the Drosophila HP1 protein, HP1a, could form droplets in vitro and that heterochromatin foci containing HP1a in early Drosophila embryos displayed properties consistent with liquid-like phase-separated states (Strom et al. 2017). In parallel, biochemical studies with human HP1 proteins found that phosphorylation of the NTE of human HP1 $\alpha$ promoted higher-order oligomerization and the formation of phaseseparated droplets (Larson et al. 2017). Binding to HP1 $\alpha$ by DNA was also shown to promote the formation of phase-separated droplets. The phase-separation behavior of HP1 $\alpha$ was compatible with at least two proposed roles of heterochromatin in cells. First, the assembly of HP1 $\alpha$ on DNA drove rapid compaction of the DNA. Second, chromatin and enzymes known to interact with HP1 $\alpha$ such as aurora B kinase could be enriched from solution in the phase-separated nPhos-HP1 $\alpha$ droplets. These observations raised the possibility that phase separation could be coupled to the chromatin compaction typically associated with heterochromatin formation. More generally, these results suggested that phase-separation processes could help to compartmentalize chromatin into active and repressed states.

Although HP1 $\alpha$ could form phase-separated droplets upon binding DNA, HP1 $\beta$ was shown to be deficient for phase-separation in the presence of DNA (Larson et al. 2017). Instead, recent work has suggested that mammalian HP $1 \beta$ requires the presence of $\mathrm{H} 3 \mathrm{~K}$ 9-methylated chromatin and the histone methylase Suvar39h1 to participate in forming phase-separated droplets (Wang et al. 2019). From a biological perspective, such biophysical differences provide a means to diversify the functions of heterochromatin.
To better understand how chromatin compaction and phase separation by HP1 are linked, we investigated the biophysical consequences of HP1 binding on chromatin. These studies were performed with Swi6 and are described below (Sanulli et al. 2019).

\section{DISORGANIZATION OF HISTONE CORE PROMOTES CHROMATIN COMPACTION INTO DROPLETS}

To understand the effect of Swi6 binding on a nucleosome, we used three complementary approaches: hydrogen-deuterium exchange-mass spectrometry (HDX-MS), nuclear magnetic resonance (NMR), and cross-linking mass spectrometry (XL-MS). The HDX-MS approach allows the determination of changes in solvent accessibility, such that increased solvent accessibility of a protein amide backbone region results in increased uptake of deuterium from solution. Given that heterochromatin is thought to be repressive and occlude access to chromatin, we hypothesized that Swi6 binding to a nucleosome would reduce uptake of deuterium by the histone proteins. Instead, we observed widespread increase in the uptake of deuterium by regions of the histone octamer core that are normally buried in the canonical nucleosome conformation. This was a surprising and counterintuitive result. We, therefore, used Methyl-TROSY NMR to further test this finding. Most commonly, NMR is used to study dynamics in proteins that are less than $\sim 30 \mathrm{kDa}$. However, methylTROSY NMR spectroscopy has made it possible to study dynamics in complexes as large as the $26 \mathrm{~S}$ proteasome 
and the nucleosome (Religa et al. 2010; Kato et al. 2011). Applying this approach indicated that binding by Swi6 results in intramolecular conformational dynamics on the msec to $\mu \mathrm{sec}$ timescale in the buried core of the nucleosome. Importantly, the changes in side-chain dynamics detected by this NMR method were consistent with the changes in backbone solvent accessibility detected by HDX-MS. In addition, using XL-MS we found changes in intrahistone cross-links upon Swi6 binding that were consistent with the changes observed by HDX-MS and NMR. Together these results indicated that binding by Swi6 to a nucleosome results in conformational reorganization of the histone core.

Additional XL-MS and NMR studies suggested that the CSD-CSD dimer contacts the nucleosome by interacting with the $\alpha 1$-helix of $\mathrm{H} 2 \mathrm{~B}$ and further implied that binding to this region of $\mathrm{H} 2 \mathrm{~B}$ may transiently unfold the helix. Based on these and previous studies described in earlier sections of this review, we proposed a model for how Swi6 could alter nucleosome conformation. In this model, Swi6 makes extensive interactions with the whole nucleosome, including the $\mathrm{H} 3$ tail, the $\mathrm{H} 2 \mathrm{~B} \alpha 1$ helix, and nucleosomal DNA. These interactions are then proposed to loosen intranucleosomal contacts, resulting in greater dynamics and exposure within the histone core (Fig. 2A,C).

To determine the significance of these conformational changes, we turned to a classic method used to study chromatin compaction by HP1 proteins (Azzaz et al. 2014; Hiragami-Hamada et al. 2016). This method uses arrays of nucleosomes, and compaction is measured by the ability to pellet the arrays in the presence of divalent ions such as $\mathrm{Mg}^{2+}$ or proteins such as HP1. We found that similar to observations with mammalian HP1 proteins, Swi6 could promote pelleting of nucleosome arrays in a concentration-dependent manner (Azzaz et al. 2014; Hiragami-Hamada et al. 2016). Interestingly, when visualized under a light microscope, the pelleted Swi6-array complexes resembled liquid droplets. The Swi6 concentration dependence for droplet formation with the arrays largely mirrored the concentration dependence for pelleting. These results provided some of the first evidence that chromatin compaction by Swi6 is coupled to phase separation. The droplets could fuse and reform spherical structures, consistent with a liquid-like nature. To assess the role of histone octamer deformation, we used sitespecific disulfide bonds to lock a region of the buried H3-H4 interface that showed major perturbations by NMR, HDX-MS, and XL-MS. Specifically, cysteine cross-links were generated between residues H3-I62C and H4-A33C within the histone octamer, and nucleosome arrays were assembled using cross-linked and uncross-linked octamers. We found that Swi6-mediated pelleting of nucleosome arrays, and corresponding droplet formation were impaired upon the introduction of these cross-links. These results indicated that the increased histone core dynamics resulting from Swi6 binding facilitate the phase separation and compaction of chromatin.

The coupling between Swi6 assembly on chromatin arrays and their compaction into phase-separated droplets gave us an opportunity to revisit the role of Swi6 oligo- merization. Here, we describe our results with a mutant in which the ARK loop within the CD is mutated to AAA (LoopX mutant). Mutating the ARK loop reduces the affinity constant for higher-order oligomerization and reduces nucleosome binding (Canzio et al. 2013). Further, the LoopX mutant results in significant defects in heterochromatin assembly, spreading, and function in $S$. pombe (Canzio et al. 2013). Surprisingly, when used under saturating conditions, we found that the LoopX mutant does not inhibit chromatin phase separation but forms larger droplets than the Swi6 wild-type (WT) protein over time. Interestingly, however, these droplets showed greater wetting behavior than the droplets formed with Swi6 WT, indicating a lower surface tension. A lower surface tension suggests that the inter-molecular interactions that enable droplet formation are weakened consistent with the reduction in the oligomerization affinity of the LoopX mutant (Alberti et al. 2019). We therefore concluded that the ability of Swi6 to compact chromatin templates into phaseseparated liquid droplets in vitro is coupled to both its ability to oligomerize and its ability to promote octamer distortion.

In $S$. pombe, distinct heterochromatin foci can be observed and Swi6 is found to accumulate at these foci (Haldar et al. 2011). To assess the correlation between the properties of Swi6-chromatin complexes in vitro and heterochromatin foci in vivo, we asked how the LoopX mutant affects heterochromatin foci in $S$. pombe. We found that replacing the wild-type Swi6 protein with the LoopX mutant reduced the number and intensity of Swi6 foci and resulted in a more diffused nuclear localization of the protein. The endogenous expression level of the LoopX protein is comparable to the WT protein, indicating that the observed defects arise from the biophysical properties of the LoopX mutant. Importantly, these results showed that the defects in heterochromatin foci observed in $S$. pombe with LoopX correlate with the instability of the LoopX-chromatin droplets in vitro.

Although the results above uncovered a functional role for the increased octamer dynamics, the question of why these dynamics are important still remained. Indeed, from a first glance, the increased accessibility and dynamics of buried histone residues appear paradoxical, given the repressive role of heterochromatin. However, these results can be explained if we consider that chromatin compaction and phase separation are linked. Substantial previous work has suggested that the formation of phase-separated assemblies relies on weak multivalent interactions (Alberti et al. 2019). We therefore propose that the transiently exposed histone residues participate in weak and multivalent interactions between nucleosomes, and that these interactions promote the compaction of chromatin into phaseseparated states.

Within the above model, we propose that an HP1 protein such as Swi6 would promote chromatin compaction in two ways: (i) by using its nucleosome deformation activity to substantially increase intrinsic histone core dynamics and accessibility, thereby increasing opportunities for weak internucleosomal interactions, and (ii) by using its oligomerization activity to bridge multiple nucleo- 


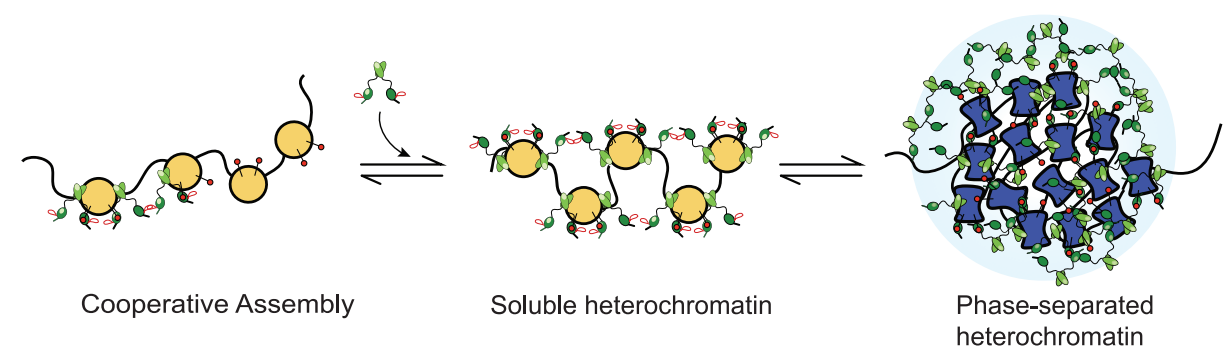

Figure 3. Model for coupling phase separation to chromatin compaction by HP1. Oligomerization by Swi6 and the transiently exposed histone core residues result in multiple weak interactions between the component molecules, which promote phase separation. The deformed nucleosomes are shown in blue.

somes and provide essential modes of multivalency (Fig. 3). Ultimately, although Swi6 increases accessibility at the level of individual nucleosomes, the net effect at the level of multiple nucleosomes is their transformation into a compact phase-separated state where the exposed histone residues become inaccessible because of participation in multivalent interactions. Additional levels of inaccessibility could arise from the specific physico-chemical properties of the phase, which may inhibit the entry of some factors and not others. Overall, such a model is consistent with previous observations of lower histone turnover in S. pombe heterochromatin and lower DNA accessibility in Drosophila heterochromatin (Aygun et al. 2013; Elgin and Reuter 2013).

\section{A BIOPHYSICAL MECHANISMS TOOL KIT: THE BENEFITS OF DIVERSITY}

It is tempting to propose a unified biophysical model for explaining the assembly of the HP1-chromatin complex. However, as described above the studies to date paint a different and more interesting picture. What is emerging is a tool kit of biophysical strategies that are used in different combinations by HP1 paralogs based on the specific chromatin context.

\section{Oligomers versus Dimers}

The higher-order oligomerization beyond dimerization observed with Swi6 suggests that oligomerization provides one mechanism to bridge nucleosomes and thereby compact the underlying chromatin. However, bridging could also be accomplished by one HP1 dimer without invoking oligomerization as has been suggested for HP1 $\beta$. It is possible that bridging via oligomerization makes the assembly process more cooperative than bridging via dimers. In contrast, bridging via dimers would make the process require less HP1 molecules per nucleosome.

\section{Mode of Nucleosome Engagement}

The CSD dimers of different HP1 proteins appear to engage different regions of the folded histone core. We speculate that these differences arise from differences in binding specificities of the respective CSD-CSD dimers.
Such differences would allow certain histone posttranslational modifications to regulate the binding of some HP1 molecules but not others. Further, the binding of nonhistone ligands to the CSD dimer could differentially affect the engagement of HP1 molecules with a nucleosome based on the relative affinities for histone versus nonhistone ligands. Finally, it is also possible that in some cases, the CSD dimer does not significantly bind the nucleosome core and the main interactions made by the HP1 dimer are with the histone $\mathrm{H} 3$ tail and the nucleosomal DNA.

\section{Octamer Deformation}

Our recent findings indicate that Swi6 binding is coupled to the deformation of the nucleosome core. We propose that such deformation is enabled in part due to the unfolding of the $\mathrm{H} 2 \mathrm{~B} \alpha 1$ helix upon binding by the CSD-CSD dimer. Analogous nucleosome deformation has not been studied for mammalian HP1 proteins. However, we speculate that the previously identified contact between the CSD-CSD dimer of mammalian HP1 proteins and the $\mathrm{H} 3 \alpha \mathrm{N}$ helix region of the nucleosome core may contribute to octamer deformation. In this context, it has been suggested that ATP-dependent chromatin remodeling by SWI/SNF family enzymes makes buried histone sites more accessible for binding by mammalian and Drosophila HP1 proteins (Lavigne et al. 2009). Interestingly SWI/SNF family remodelers have been suggested to alter nucleosome conformation (Fan et al. 2004; Sinha et al. 2017). Building on these studies we speculate that in some contexts, HP1 proteins may collaborate with ATP-dependent remodelers to drive octamer deformation.

\section{Phase Separation}

We propose that the different abilities of HP1 proteins to phase-separate in the presence of chromatin adds to the diversity of HP1-mediated heterochromatin. Thus, we can imagine that in contrast to Swi6, some HP1 paralogs may form phases with chromatin by relying solely on the multivalency provided by their oligomerization and not via octamer deformation. Other HP1 proteins may compact chromatin via nucleosome bridging but not cause phase separation. Overall, we imagine a large range of different 
types of interaction networks between the HP1 proteins and chromatin within and outside phase-separated heterochromatin states.

The biophysical strategies described above provide fertile ground for conceptualizing how the core HP1-chromatin complex can participate in diverse nuclear functions. For example, it is possible that HP1-heterochromatin at the nuclear lamina exists in a phase-separated state. Because of the nature of the multivalent interactions, such a phase-separated state could possess the viscoelasticity required to withstand mechanical forces exerted on the nucleus. At another extreme, it is possible that heterochromatin that contains a non-phase-separated state of the HP1-chromatin complex is more responsive to developmental cues. We look forward to future studies that will shed light on additional biophysical strategies that are used in the biological regulation of heterochromatin function.

\section{ACKNOWLEDGMENTS}

We thank members of the Narlikar laboratory for the many stimulating discussions over the past decade that have helped in formulating this review. This work was supported by the National Institutes of Health (NIH) grant R35 GM127020 to G.J.N.

\section{REFERENCES}

Alberti S, Gladfelter A, Mittag T. 2019. Considerations and challenges in studying liquid-liquid phase separation and biomolecular condensates. Cell 176: 419-434. doi:10.1016/j.cell .2018 .12 .035

Allshire RC, Madhani HD. 2018. Ten principles of heterochromatin formation and function. Nat Rev Mol Cell Biol 19: 229244. doi:10.1038/nrm.2017.119

Aygün O, Mehta S, Grewal SI. 2013. HDAC-mediated suppression of histone turnover promotes epigenetic stability of heterochromatin. Nat Struct Mol Biol 20: 547-554. doi:10.1038/ nsmb. 2565

Azzaz AM, Vitalini MW, Thomas AS, Price JP, Blacketer MJ, Cryderman DE, Zirbel LN, Woodcock CL, Elcock AH, Wallrath LL, et al. 2014. Human heterochromatin protein $1 \alpha$ promotes nucleosome associations that drive chromatin condensation. J Biol Chem 289: 6850-6861. doi:10.1074/jbc .M113.512137

Bannister AJ, Zegerman P, Partridge JF, Miska EA, Thomas JO, Allshire RC, Kouzarides T. 2001. Selective recognition of methylated lysine 9 on histone H3 by the HP1 chromo domain. Nature 410: 120-124. doi:10.1038/35065138

Brasher SV, Smith BO, Fogh RH, Nietlispach D, Thiru A, Nielsen PR, Broadhurst RW, Ball LJ, Murzina NV, Laue ED. 2000. The structure of mouse HP1 suggests a unique mode of single peptide recognition by the shadow chromo domain dimer. EMBO J 19: 1587-1597. doi:10.1093/emboj/19.7 .1587

Canzio D, Chang EY, Shankar S, Kuchenbecker KM, Simon MD, Madhani HD, Narlikar GJ, Al-Sady B. 2011. Chromodomain-mediated oligomerization of HP1 suggests a nucleosome-bridging mechanism for heterochromatin assembly. Mol Cell 41: 67-81. doi:10.1016/j.molcel.2010.12.016

Canzio D, Liao M, Naber N, Pate E, Larson A, Wu S, Marina DB, Garcia JF, Madhani HD, Cooke R, et al. 2013. A conformational switch in HP1 releases auto-inhibition to drive heterochromatin assembly. Nature 496: 377-381. doi:10.1038/ nature 12032
Canzio D, Larson A, Narlikar GJ. 2014. Mechanisms of functional promiscuity by HP1 proteins. Trends Cell Biol 24: 377 386. doi:10.1016/j.tcb.2014.01.002

Cowieson NP, Partridge JF, Allshire RC, McLaughlin PJ. 2000. Dimerisation of a chromo shadow domain and distinctions from the chromodomain as revealed by structural analysis. Curr Biol 10: 517-525. doi:10.1016/S0960-9822 (00)00467-X

Dawson MA, Bannister AJ, Gottgens B, Foster SD, Bartke T, Green AR, Kouzarides T. 2009. JAK2 phosphorylates histone H3Y41 and excludes HP1 $\alpha$ from chromatin. Nature 461: 819822. doi: $10.1038 /$ nature 08448

Eissenberg JC, Elgin SC. 2014. HP1 $\alpha$ : a structural chromosomal protein regulating transcription. Trends Genet 30: 103-110. doi:10.1016/j.tig.2014.01.002

Eissenberg JC, James TC, Foster-Hartnett DM, Hartnett T, Ngan V, Elgin SC. 1990. Mutation in a heterochromatinspecific chromosomal protein is associated with suppression of position-effect variegation in Drosophila melanogaster. Proc Natl Acad Sci 87: 9923-9927. doi:10.1073/pnas.87.24 .9923

Elgin SCR, Reuter G. 2013. Position-effect variegation, heterochromatin formation, and gene silencing in Drosophila. Cold Spring Harb Perspect Biol 5: a017780. doi:10.1101/cshper spect.a017780

Fan HY, Narlikar GJ, Kingston RE. 2004. Noncovalent modification of chromatin: different remodeled products with different ATPase domains. Cold Spring Harb Symp Quant Biol 69: 183-192. doi:10.1101/sqb.2004.69.183

Grewal SI, Jia S. 2007. Heterochromatin revisited. Nat Rev Genet 8: 35 -46. doi: $10.1038 / \mathrm{nrg} 2008$

Haldar S, Saini A, Nanda JS, Saini S, Singh J. 2011. Role of Swi6/HP1 self-association-mediated recruitment of Clr4/ Suv39 in establishment and maintenance of heterochromatin in fission yeast. $J$ Biol Chem 286: 9308-9320. doi:10.1074/jbc .M110.143198

Heitz E. 1928. Das Heterochromatin der Moose. Jahrb Wiss Bot 69: $762-818$.

Hiragami-Hamada K, Shinmyozu K, Hamada D, Tatsu Y, Uegaki K, Fujiwara S, Nakayama J. 2011. N-terminal phosphorylation of HP1 $\alpha$ promotes its chromatin binding. Mol Cell Biol 31: 1186-1200. doi:10.1128/MCB.01012-10

Hiragami-Hamada K, Soeroes S, Nikolov M, Wilkins B, Kreuz S, Chen C, De La Rosa-Velázquez IA, Zenn HM, Kost N, Pohl W, et al. 2016. Dynamic and flexible H3K9me3 bridging via HP1 $\beta$ dimerization establishes a plastic state of condensed chromatin. Nat Commun 7: 11310. doi:10.1038/ ncomms 11310

Isaac RS, Sanulli S, Tibble R, Hornsby M, Ravalin M, Craik CS, Gross JD, Narlikar GJ. 2017. Biochemical basis for distinct roles of the heterochromatin proteins Swi6 and Chp2. J Mol Biol 429: 3666-3677. doi:10.1016/j.jmb.2017.09.012

Jacobs SA, Khorasanizadeh S. 2002. Structure of HP1 chromodomain bound to a lysine 9-methylated histone H3 tail. Science 295: 2080-2083. doi:10.1126/science.1069473

Jacobs SA, Taverna SD, Zhang Y, Briggs SD, Li J, Eissenberg JC, Allis CD, Khorasanizadeh S. 2001. Specificity of the HP1 chromo domain for the methylated N-terminus of histone H3. EMBO J 20: 5232-5241. doi:10.1093/emboj/20.18 .5232

James TC, Elgin SC. 1986. Identification of a nonhistone chromosomal protein associated with heterochromatin in Drosophila melanogaster and its gene. Mol Cell Biol 6: 3862-3872. doi:10.1128/MCB.6.11.3862

James TC, Eissenberg JC, Craig C, Dietrich V, Hobson A, Elgin SC. 1989. Distribution patterns of HP1, a heterochromatinassociated nonhistone chromosomal protein of Drosophila. Eur J Cell Biol 50: 170-180.

Janssen A, Colmenares SU, Karpen GH. 2018. Heterochromatin: guardian of the genome. Anпu Rev Cell Dev Biol 34: 265-288. doi:10.1146/annurev-cellbio-100617-062653 
Jost KL, Bertulat B, Cardoso MC. 2012. Heterochromatin and gene positioning: inside, outside, any side? Chromosoma 121: 555-563. doi:10.1007/s00412-012-0389-2

Kang J, Chaudhary J, Dong H, Kim S, Brautigam CA, Yu H. 2011. Mitotic centromeric targeting of HP1 and its binding to Sgol are dispensable for sister-chromatid cohesion in human cells. Mol Biol Cell 22: 1181-1190. doi:10.1091/mbc.e11-010009

Kato H, van Ingen H, Zhou B-R, Feng H, Bustin M, Kay LE, Bai Y. 2011. Architecture of the high mobility group nucleosomal protein 2-nucleosome complex as revealed by methyl-based NMR. Proc Natl Acad Sci 108: 12283-12288. doi:10.1073/ pnas. 1105848108

Kilic S, Felekyan S, Doroshenko O, Boichenko I, Dimura M, Vardanyan H, Bryan LC, Arya G, Seidel CAM, Fierz B. 2018. Single-molecule FRET reveals multiscale chromatin dynamics modulated by HP1 $\alpha$. Nat Comm 9: 235. doi:10.1038/ s41467-017-02619-5

Lachner M, O'Carroll D, Rea S, Mechtler K, Jenuwein T. 2001. Methylation of histone H3 lysine 9 creates a binding site for HP1 proteins. Nature 410: 116-120. doi:10.1038/ 35065132

Larson AG, Elnatan D, Keenen MM, Trnka MJ, Johnston JB, Burlingame AL, Agard DA, Redding S, Narlikar GJ. 2017. Liquid droplet formation by HP1 $\alpha$ suggests a role for phase separation in heterochromatin. Nature 547: 236-240. doi:10 $.1038 /$ nature 22822

Lavigne M, Eskeland R, Azebi S, Saint-Andre V, Jang SM, Batsche E, Fan HY, Kingston RE, Imhof A, Muchardt C. 2009. Interaction of HP1 and Brg1/Brm with the globular domain of histone $\mathrm{H} 3$ is required for HP1-mediated repression. PLoS Genet 5: e1000769. doi:10.1371/journal .pgen.1000769

Lechner MS, Schultz DC, Negorev D, Maul GG, Rauscher FJ III. 2005. The mammalian heterochromatin protein 1 binds diverse nuclear proteins through a common motif that targets the chromoshadow domain. Biochem Biophys Res Commun 331: 929 937. doi:10.1016/j.bbrc.2005.04.016

Luger K, Mäder AW, Richmond RK, Sargent DF, Richmond TJ. 1997. Crystal structure of the nucleosome core particle at $2.8 \AA$ resolution. Nature 389: 251-260. doi:10.1038/38444

Machida S, Takizawa Y, Ishimaru M, Sugita Y, Sekine S, Nakayama JI, Wolf M, Kurumizaka H. 2018. Structural basis of heterochromatin formation by human HP1. Mol Cell 69: 385-397.e388. doi:10.1016/j.molcel.2017.12.011

Martienssen R, Moazed D. 2015. RNAi and heterochromatin assembly. Cold Spring Harb Perspect Biol 7: a019323. doi:10.1101/cshperspect.a019323

Meehan RR, Kao CF, Pennings S. 2003. HP1 binding to native chromatin in vitro is determined by the hinge region and not by the chromodomain. EMBO J 22: 3164-3174. doi:10.1093/ emboj/cdg306

Mendez DL, Kim D, Chruszcz M, Stephens GE, Minor W, Khorasanizadeh S, Elgin SC. 2011. The HP1a disordered C terminus and chromo shadow domain cooperate to select target peptide partners. Chembiochem 12: 1084-1096. doi:10.1002/ cbic. 201000598

Mendez DL, Mandt RE, Elgin SC. 2013. Heterochromatin Protein 1a (HP1a) partner specificity is determined by critical amino acids in the chromo shadow domain and C-terminal extension. J Biol Chem 288: 22315-22323. doi:10.1074/jbc .M113.468413

Mishima Y, Watanabe M, Kawakami T, Jayasinghe CD, Otani J, Kikugawa Y, Shirakawa M, Kimura H, Nishimura O, Aimoto $\mathrm{S}$, et al. 2013. Hinge and chromoshadow of HP1 $\alpha$ participate in recognition of $\mathrm{K} 9$ methylated histone $\mathrm{H} 3$ in nucleosomes. $J$ Mol Biol 425: 54-70. doi:10.1016/j.jmb.2012.10.018

Muchardt C, Guilleme M, Seeler JS, Trouche D, Dejean A, Yaniv M. 2002. Coordinated methyl and RNA binding is required for heterochromatin localization of mammalian HP1 $\alpha$. EMBO Rep 3: 975-981. doi:10.1093/embo-reports/kvf194
Nicetto D, Zaret KS. 2019. Role of H3K9me3 heterochromatin in cell identity establishment and maintenance. Curr Opin Genet Dev 55: 1-10. doi:10.1016/j.gde.2019.04.013

Nielsen PR, Nietlispach D, Mott HR, Callaghan J, Bannister A, Kouzarides T, Murzin AG, Murzina NV, Laue ED. 2002. Structure of the HP1 chromodomain bound to histone H3 methylated at lysine 9. Nature 416: 103-107. doi:10.1038/ nature 722

Nishibuchi G, Machida S, Osakabe A, Murakoshi H, HiragamiHamada K, Nakagawa R, Fischle W, Nishimura Y, Kurumizaka H, Tagami H, et al. 2014. N-terminal phosphorylation of HP1 $\alpha$ increases its nucleosome-binding specificity. Nucleic Acids Res 42: 12498-12511. doi:10.1093/nar/gku995

Rea S, Eisenhaber F, O'Carroll D, Strahl BD, Sun Z-W, Schmid M, Opravil S, Mechtler K, Ponting CP, Allis CD, et al. 2000. Regulation of chromatin structure by site-specific histone $\mathrm{H} 3$ methyltransferases. Nature 406: 593-599. doi:10.1038/ 35020506

Religa TL, Sprangers R, Kay LE. 2010. Dynamic regulation of archaeal proteasome gate opening as studied by TROSY NMR. Science 328: 98-102. doi:10.1126/science .1184991

Reyes-Turcu FE, Grewal SI. 2012. Different means, same endheterochromatin formation by RNAi and RNAi-independent RNA processing factors in fission yeast. Curr Opin Genet Dev 22: 156-163. doi:10.1016/j.gde.2011.12.004

Richart AN, Brunner CI, Stott K, Murzina NV, Thomas JO. 2012. Characterization of chromoshadow domain-mediated binding of heterochromatin protein $1 \alpha(\mathrm{HP} 1 \alpha)$ to histone H3. J Biol Chem 287: 18730-18737. doi:10.1074/jbc.M111 .337204

Sadaie M, Kawaguchi R, Ohtani Y, Arisaka F, Tanaka K, Shirahige K, Nakayama J. 2008. Balance between distinct HP1 family proteins controls heterochromatin assembly in fission yeast. Mol Cell Biol 28: 6973-6988. doi:10.1128/MCB .00791-08

Sanulli S, Trnka MJ, Dharmarajan V, Tibble RW, Pascal BD, Burlingame AL, Griffin PR, Gross JD, Narlikar GJ. 2019. HP1 reshapes nucleosome core to promote phase separation of heterochromatin. Nature 575: 390-394. doi:10.1038/ s41586-019-1669-2

Schuettengruber B, Bourbon HM, Di Croce L, Cavalli G. 2017. Genome regulation by polycomb and trithorax: 70 years and counting. Cell 171: 34-57. doi:10.1016/j.cell.2017.08.002

Simon JA, Kingston RE. 2009. Mechanisms of polycomb gene silencing: knowns and unknowns. Nat Rev Mol Cell Biol 10: 697-708. doi:10.1038/nrm2763

Sinha KK, Gross JD, Narlikar GJ. 2017. Distortion of histone octamer core promotes nucleosome mobilization by a chromatin remodeler. Science 355: eaaa3761. doi:10.1126/science .aaa3761

Smothers JF, Henikoff S. 2000. The HP1 chromo shadow domain binds a consensus peptide pentamer. Curr Biol 10: 27-30. doi:10.1016/S0960-9822(99)00260-2

Stephens AD, Banigan EJ, Marko JF. 2019. Chromatin's physical properties shape the nucleus and its functions. Curr Opin Cell Biol 58: 76-84. doi:10.1016/j.ceb.2019.02.006

Strom AR, Emelyanov AV, Mir M, Fyodorov DV, Darzacq X, Karpen GH. 2017. Phase separation drives heterochromatin domain formation. Nature 547: 241-245. doi:10 .1038 /nature22989

Sugimoto K, Yamada T, Muro Y, Himeno M. 1996. Human homolog of Drosophila heterochromatin-associated protein 1 (HP1) is a DNA-binding protein which possesses a DNAbinding motif with weak similarity to that of human centromere protein C (CENP-C). J Biochem 120: 153-159. doi:10 .1093/oxfordjournals.jbchem.a021378

Thiru A, Nietlispach D, Mott HR, Okuwaki M, Lyon D, Nielsen PR, Hirshberg M, Verreault A, Murzina NV, Laue ED. 2004. Structural basis of HP1/PXVXL motif peptide interactions and HP1 localisation to heterochromatin. EMBO J 23: 489-499. doi:10.1038/sj.emboj. 7600088 
Wang G, Ma A, Chow CM, Horsley D, Brown NR, Cowell IG, Singh PB. 2000. Conservation of heterochromatin protein 1 function. Mol Cell Biol 20: 6970-6983. doi:10.1128/MCB.20 18.6970-6983.2000

Wang L, Gao Y, Zheng X, Liu C, Dong S, Li R, Zhang G, Wei Y, $\mathrm{Qu} \mathrm{H}, \mathrm{Li}$ Y, et al. 2019. Histone modifications regulate chromatin compartmentalization by contributing to a phase separation mechanism. Mol Cell 76: 646-659.e6. doi:10.1016/j .molcel.2019.08.019
Yamada T, Fukuda R, Himeno M, Sugimoto K. 1999. Functional domain structure of human heterochromatin protein HP1 $(\mathrm{Hs} \alpha)$ : involvement of internal DNA-binding and C-terminal self-association domains in the formation of discrete dots in interphase nuclei. J Biochem 125: 832-837. doi:10.1093/ oxfordjournals.jbchem.a022356

Yu J-R, Lee CH, Oksuz O, Stafford JM, Reinberg D. 2019. PRC2 is high maintenance. Genes Dev 33: 903-935. doi:10.1101/ $\operatorname{gad} .325050 .119$ 


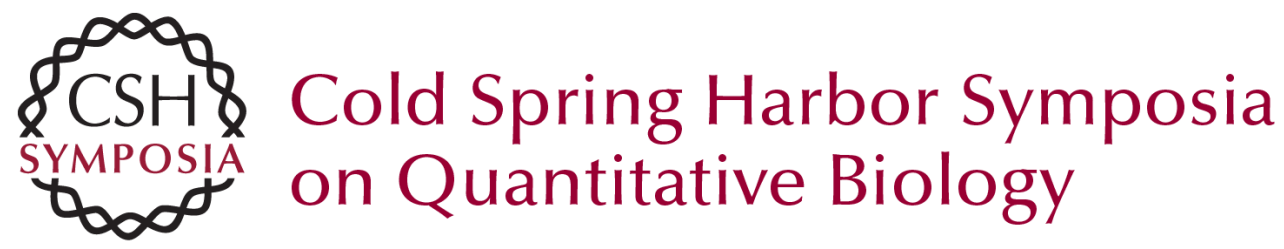

\title{
Biophysical Properties of HP1-Mediated Heterochromatin
}

\author{
Serena Sanulli, John D. Gross and Geeta J. Narlikar
}

Cold Spring Harb Symp Quant Biol published online June 3, 2020

Access the most recent version at doi:10.1101/sqb.2019.84.040360

\begin{aligned} & $\mathbf{P}<\mathbf{P}$ Published online June 3,2020 in advance of the print journal. \\ & $\begin{array}{r}\text { Creative } \\ \text { Commons } \\ \text { License }\end{array} \begin{array}{l}\text { This article is distributed under the terms of the } \\ \text { http://creativecommons.org/licenses/by-nc/4.0/, which permits reuse and } \\ \text { redistribution, except for commercial purposes, provided that the original } \\ \text { author and source are credited. }\end{array} \\ & \begin{aligned} \text { Email Alerting } \\ \text { Service }\end{aligned} \begin{array}{l}\text { Receive free email alerts when new articles cite this article - sign up in } \\ \text { the box at the top right corner of the article or click here. }\end{array} \\ &$\hline\end{aligned}

Advance online articles have been peer reviewed and accepted for publication but have not yet appeared in the paper journal (edited, typeset versions may be posted when available prior to final publication). Advance online articles are citable and establish publication priority; they are indexed by PubMed from initial publication. Citations to Advance online articles must include the digital object identifier (DOIs) and date of initial publication.

To subscribe to Cold Spring Harbor Symposia on Quantitative Biology go to: http://symposium.cshlp.org/subscriptions 